\title{
THE
}

6-1-1993

\section{Antiferromagnetic Triangular Ising Model: Critical Behavior of the Ground State}

Henk W.J. Blöte

M. P. Nightingale

University of Rhode Island, nightingale@uri.edu

Follow this and additional works at: https://digitalcommons.uri.edu/phys_facpubs

Terms of Use

All rights reserved under copyright.

\section{Citation/Publisher Attribution}

Blöte, H. W. J. \& Nightingale, M. P. (1993). Antiferromagnetic triangular Ising model: Critical behavior of the ground state. Physical Review B, 47(22), 15046-15059. doi: 10.1103/PhysRevB.47.15046

Available at: http://dx.doi.org/10.1103/PhysRevB.47.15046

This Article is brought to you for free and open access by the Physics at DigitalCommons@URI. It has been accepted for inclusion in Physics Faculty Publications by an authorized administrator of DigitalCommons@URI. For more information, please contact digitalcommons-group@uri.edu. 


\title{
Antiferromagnetic triangular Ising model: Critical behavior of the ground state
}

\author{
Henk W. J. Blöte \\ Laboratorium voor Technische Natuurkunde, Technische Universiteit Delft, Postbus 5046, 2600 GA Delft, The Netherlands \\ M. Peter Nightingale \\ Physics Department, University of Rhode Island, Kingston, Rhode Island 02881 \\ (Received 11 June 1992; revised manuscript received 2 March 1993)
}

\begin{abstract}
The critical ground state of the antiferromagnetic triangular Ising model is studied with finite-size scaling, with the use of exact techniques and numerical transfer-matrix methods. Exact solution of the model in zero field yields a conformal anomaly equal to 1 , and values of critical exponents. For nonzero fields, dominant eigenvalues of the transfer matrix are computed for systems with linear dimensions up to 27 with the use of an efficient coding of spin states as a system of nonintersecting strings. The results are in perfect agreement with the Gaussian-model-Coulomb-gas scenario proposed by Nienhuis et al. for this model. Quantitative agreement is found with the predictions for exponents of spin-wave and vortex operators. Furthermore, we locate the field-induced Kosterlitz-Thouless transition to a longrange-ordered state at a field $H_{c}=0.266 \pm 0.010$.
\end{abstract}

\section{INTRODUCTION}

Three mutually interacting spins can be pairwise parallel, but not pairwise antiparallel. This leads to an asymmetry between the ferromagnetic and antiferromagnetic spin models on the triangular lattice. For instance, consider a triangular Ising model with equal nearestneighbor couplings $K$ described by the reduced Hamiltonian

$$
-\frac{\mathscr{H}_{\text {Ising }}}{k T}=K \sum_{\langle i, j\rangle} s_{i} s_{j}+H \sum_{k} s_{k},
$$

where $\langle i, j\rangle$ indicates summation over pairs of nearestneighbor sites. The exact solution of this $\operatorname{model}^{1}$ in zero reduced field $H$ shows that there is no antiferromagnetic phase transition at nonzero temperature. In this paper we shall deal almost exclusively with the $K \rightarrow-\infty$ limit of this model, i.e., the zero-temperature triangular antiferromagnetic Ising model or Ising model for short. The corresponding ground state has interesting properties: It is infinitely degenerate and carries a nonzero residual entropy density. It is in fact a critical state, as follows, e.g., from the algebraic decay of correlations. ${ }^{2}$ Furthermore, there exists an exact mapping onto the triangular solidon-solid (SOS) model, which describes the equilibrium shape of a cubic crystal ${ }^{3}$ near its $[1,1,1]$ corner. An exact solution $^{4,3}$ has shown that this system undergoes a commensurate-incommensurate transition when finite differences between the infinite couplings are introduced in the three lattice directions of the original Ising model, but here we shall consider only the isotropic model.

The SOS model is defined on a triangular lattice $\mathcal{L}$, consisting of three interpenetrating triangular sublattices $\mathcal{L}_{l}, l=0,1,2$. The height of the SOS surface at site $k \in \mathcal{L}$ is integral and denoted by $h_{k}$. The $h_{k}$ have the following restrictions: (i) The height difference of no nearestneighbor pair of sites is to exceed two units; (ii) on sublat- tice $\mathcal{L}_{1}$, the height variables $\bmod 3$ equal $l$. The reduced Hamiltonian is

$$
\begin{aligned}
-\frac{\mathcal{H}_{\mathrm{SOS}}}{k T}= & K_{\infty} \sum_{\langle i, j\rangle} \Theta\left(\left|h_{i}-h_{j}\right|-3\right) \\
& +H_{\infty} \sum_{l=0}^{2} \sum_{k \in \mathcal{L}_{l}}\left(1-\delta_{\left(h_{k} \bmod 3\right), l}\right) \\
& +H \sum_{k \in \mathcal{L}}(-1)^{h_{k}},
\end{aligned}
$$

where $\Theta$ is the step function, in the limit $K_{\infty}=H_{\infty} \rightarrow-\infty$. The first two terms impose the restrictions described above. The last two terms are periodic potentials on the height variables with periods 2 and 3, so that the system is invariant under global changes of the heights generated by the transformation $h_{k} \rightarrow h_{k} \pm 6$. However, cyclic permutations of the sublattices involving a translation by one lattice unit change the height variables by one, which corresponds with a symmetry under global changes of height by one unit. This symmetry holds only when $H=0$ because the last term in Eq. (2) changes sign under this operation. For $H \neq 0$ the global symmetry of Eq. (2) is over two units of height. For completeness we review the transcription ${ }^{4}$ of the Ising spin configurations to SOS height configurations. A spin configuration is represented as a tiling of the plane by $60^{\circ}$ rhombi whose edges connect nearest-neighbor spins of opposite signs. Choose an elementary up-triangle [see Fig. 1(a)]. Following the edges counterclockwise, the SOS height increases by one unit between antiparallel spins and decreases by two units between parallel spins. The same holds for the down-triangles if one follows the edges clockwise instead. Note that with a single spin and a single height fixed, this is a one-to-one correspondence (disregarding dislocations, as further qualified below).

The phase diagram of the zero-temperature triangular Ising antiferromagnet in a magnetic field follows from the 
exact mapping of the Ising model, Eq. (1), to the SOS model, Eq. (2), and a subsequent standard, but not rigorously justified renormalization-group mapping ${ }^{5-8}$ to the Gaussian model. This analysis predicts that this Ising model is in the universality class of the Gaussian model with Hamiltonian

$-\frac{\mathscr{H}}{k T}=\frac{2 \pi}{T_{R}} \sum_{\langle i, j\rangle}\left(h_{i}-h_{j}\right)^{2}+\sum_{p} w_{p} \sum_{i} \cos \frac{2 \pi h_{i}}{p}$.

Here the heights $h_{i}$ are continuous variables. Spin-wave terms given by the double sum have the following origins: (i) The term with $p=1$ reflects the discreteness of the height variables in the original SOS model, i.e., the periodicity of Eq. (2) with global changes of height by 1 when $H=0$; (ii) a nonzero field $H$ lowers the symmetry of Eq. (2) under global changes of height to two units, and thus generates a $p=2$ term, with $w_{2} \propto H$; and (iii) $p=3$ or $p=6$ terms are absent for the uniform model given in Eq. (1), but they can be introduced by considering nearest-neighbor couplings $(p=3)$ or magnetic fields $(p=6)$ that are sublattice dependent.

For the SOS model the correlation function $\boldsymbol{G}(\boldsymbol{r})=\left\langle\left(h_{0}-h_{r}\right)^{2}\right\rangle$ of two height variables at a distance $r$ can be obtained from the energy-energy correlation function of the triangular Ising model, ${ }^{4}$ which is known exactly. ${ }^{2}$ The result ${ }^{4}$ for the zero-field SOS model, Eq. (2), is

$$
G(r) \simeq \frac{9}{\pi^{2}} \ln r
$$

This is the key to the identification ${ }^{3}$ of the temperature $T_{R}$ of the Gaussian model onto which the $H=0$ Ising model maps under renormalization. That is, for the Gaussian model, Eq. (3), one readily finds the asymptotic long-distance behavior of $G(r)$ for $w_{p}=0$ :

$$
G(r) \simeq \frac{T_{R}}{2 \pi^{2}} \ln r .
$$

The amplitude of the correlation function in Eq. (4) is invariant under renormalization. Equating the amplitudes of both exact results yields $T_{R}=18$ at $H=0$.

The subject of this paper is the predicted nonuniversal behavior of the critical ground state of the triangular Ising antiferromagnet in a finite and nonvanishing range of fields $H$ terminating in a Kosterlitz-Thouless transition ${ }^{9}$ to a state with long-range order. This phase transition corresponds to a roughening transition ${ }^{5,6,10}$ of the SOS model, Eq. (2), the rough phase occurring for $H$ small in magnitude. The existence of such a Kosterlitz-Thouless transition was recently confirmed ${ }^{11}$ by means of finite-size scaling.

For completeness we mention that Nienhuis, Hilhorst, and Blöte ${ }^{3}$ qualitatively derived the phase diagram as a function of the external field and additional next-nearestneighbor couplings. The topology of this phase diagram derives from the identification of the relevant operators contained in the Ising model with SOS operators, and predictions for the critical exponents in zero field and at the roughening transition. That is, again following the standard procedure, once $T_{R}$ is known, the anomalous dimensions can be derived from the Gaussian model. The term in the Gaussian model proportional to $w_{p}$, i.e., the spin-wave operator of period $p$, has a critical dimension

$$
X_{p}^{(s)}=\frac{T_{R}}{2 p^{2}} .
$$

Consequently, the spin-wave operator with period 1 , corresponding to the discrete character of the SOS heights, is irrelevant for $T_{R}=18$. The same holds for the $p=2$ spin-wave operator, corresponding to the uniform magnetic field $H$. Hence the prediction that a sufficiently small magnetic field does not destroy the critical state of Eqs. (1) and (2). ${ }^{3}$ The Gaussian temperature $T_{R}$ decreases with increasing $H$, until at $T_{R}=16$ the $p=2$ operator become relevant.

For $p \geq 3$ the spin-wave operators are relevant at $H=0$. The $p=3$ operator corresponds to a periodic modulation of the Ising couplings, which indeed introduces a phase transition to a long-range-ordered state. ${ }^{3}$ The most relevant spin-wave operator has $p=6$; this is the longest period compatible with the Ising representation of the model. It corresponds to a staggered field acting on the Ising spins, e.g., positive on one sublattice and negative on the two other ones. In zero uniform field its dimension is, according to Eq. (6), $X_{6}^{(s)}=\frac{1}{4}$, which agrees with Stephenson's result ${ }^{2} \eta=\frac{1}{2}$ for the spin-spin correlation function because of the identification $T_{R}=18$ at $H=0$.

For nonzero Ising temperatures elementary triangles with three spins of the same sign are no longer frozen out. As illustrated in Fig. 1, they produce in the SOS surface screw dislocations with Burgers vectors of \pm 6 units. ${ }^{3}$ More generally we shall consider $q$-fold dislocations. In

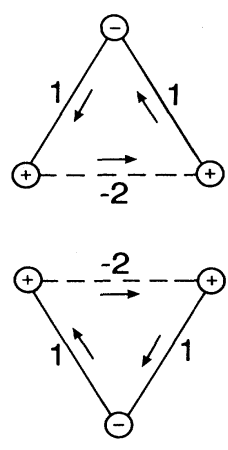

(a)

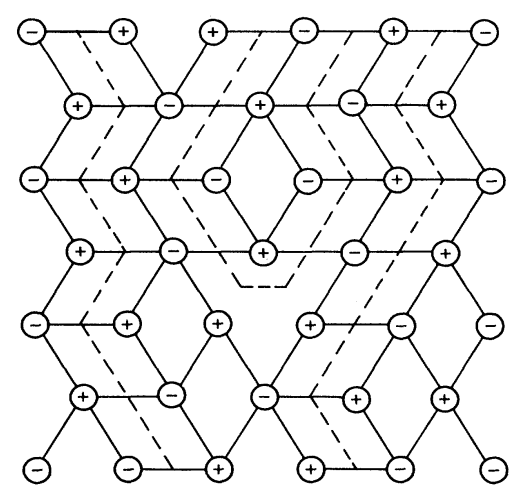

(b)
FIG. 1. Ising, tiling, string, and SOS representations of a configuration including a vortex. (a) The correspondence between Ising spin configurations and SOS height differences. (b) The tiling representation is obtained by connecting antiparallel neighbor spins. The elementary faces of the lattice combine into rhombuses with three possible orientations. The string representation follows by connecting the midpoints of the horizontal edges of the rhombuses (dashed lines). An elementary Ising excitation consists of a triangle of parallel spins, and corresponds with a triangular tile having a linear size twice that of an elementary face, and with a change of the number of strings by two. Since each string corresponds with a change of height of three units, the corresponding vortices have strength $q= \pm 6$. 
the Gaussian model the corresponding $q$-fold dislocation or vortex operators have anomalous dimensions

$$
X_{q}^{(v)}=\frac{q^{2}}{2 T_{R}} .
$$

Equivalently one can formulate these operators in terms of the Coulomb gas. The spin-wave operators in the Gaussian model correspond with electric charges $e$ in the Coulomb gas and vortex operators with magnetic charges $m$. The Coulomb gas is described by a reduced Hamiltonian

$$
\begin{aligned}
-\frac{\mathcal{H}_{c g}}{k T}=\sum_{\langle j, k\rangle}\{ & {\left[\frac{1}{g_{c g}} e_{j} e_{k}+g_{c g} m_{j} m_{k}\right] G\left(\mathbf{r}_{j}-\mathbf{r}_{k}\right) } \\
& \left.+2 i e_{j} m_{k} \Phi\left(\mathbf{r}_{j}-\mathbf{r}_{k}\right)\right\}+\sum_{l} \mu\left(e_{l}, m_{l}\right),
\end{aligned}
$$

where $g_{c g}$ is the coupling constant and $\mu(e, m)$ is the chemical potential of a gas particle with charges as indicated. In particular we consider the limit $\mu(e, m) \rightarrow-\infty$ where all particle densities vanish; this limit is supposed to be approached under renormalization. The long-range asymptotic behavior of the potentials between the charges is determined by $\exp [G(x, y)+i \Phi(x, y)]$ $=-(x+i y) / a$, where $a$ is a short-distance cutoff parameter. For more details, see the review given in Ref. 7 .

It is natural to fix the scale of the charges such that the largest spin-wave period $p=6$ in Eq. (3) corresponds to an electric charge $e=1$ in the Coulomb gas. The anomalous dimension associated with electromagnetic charges $\pm(e, m)$ is

$$
X_{e, m}=\frac{e^{2}}{2 g_{c g}}+\frac{g_{c g} m^{2}}{2} .
$$

The requirement $X_{1,0}=X_{6}^{(s)}=\frac{1}{4}$ shows that $g_{c g}=36 / T_{R}$ or $g_{c g}=2$ corresponds to $H=0$ in Eqs. (1) and (2). Indeed, the elementary vortex of strength 6 , which has $X_{6}^{(v)}=1$, then corresponds to the elementary magnetic charge $m=1: X_{0,1}=1$. Thus, the correspondence between the Gaussian model, Eq. (3), and the Coulomb gas, Eq. (8), is determined by

$$
g_{c g}=36 / T_{R}, \quad e=6 / p, \quad m=q / 6 .
$$

In the following we adhere to the Gaussian language for reasons of easy compatibility with Ref. 3 .

The rest of this paper is organized as follows. With both exact (see Sec. II) and numerical (see Sec. III) calculations, we corroborate the renormalization-group scenario outlined above. Section III A contains exact results for the conformal anomaly $c$ and spin-wave exponent $X_{6}^{(s)}$ for the zero-temperature triangular Ising antiferromagnet in zero field. Exact results for the vortex exponent $X_{2}^{(v)}$ are derived in Sec. II B. The numerical analysis is based on the relation between the eigenvalue spectrum of the transfer matrix and the vortex and spinwave exponents. Thus the calculation of these eigenvalues reveals the scaling behavior of the model. Section III A is devoted to numerical determination of $H_{c}$, the value of the field at which the Kosterlitz-Thouless transition takes place. Numerical results for the conformal anomaly and critical dimensions for nonzero magnetic fields $(H \neq 0)$ can be found in Secs. III B and III C, while a discussion and summary of the results is presented in Sec. IV. Finally, Appendixes A and B contain technical details of the exact and numerical calculations, respectively.

\section{EXACT RESULTS}

We consider finite systems of rectangular shape with periodic boundary conditions. One side of the rectangle is formed by the bases of $L$ elementary triangles. This defines the horizontal or $L$ direction. The other side of the rectangle runs in the vertical or $M$ direction and has the length of $M$ times the height of an elementary triangle. We consider the limit $M \rightarrow \infty$.

An Ising spin configuration can be represented by an SOS surface, as mentioned in the Introduction, and before presenting our results we discuss some complications of this representation. First we consider the introduction of defect lines in the associated SOS surface by the use of periodic boundary conditions. If one moves to the right along the horizontal direction, a pair of opposite spins, as we recall, corresponds to a step up of unit height in the SOS surface, while a pair of like spins is two steps down. These possibilities are represented respectively by edges and short diagonals of rhombi in the tiling representation. A convenient way of representing the SOS surface, in particular for numerical purposes, is by strings running in the vertical direction. As illustrated in Fig. 1, these strings are defined by drawing lines connecting the middles of horizontal edges of rhombi. The sum of these height differences along a loop circling the lattice once in the horizontal direction equals

$$
q=3 N_{s}-2 L
$$

the strength of the defect, for a system with $N_{s}$ strings. This sum vanishes only if there are precisely $2 L / 3$ strings, i.e., pairs of opposite nearest-neighbor spins, a condition requiring $L$ to be a multiple of 3 . Violation of this requirement corresponds to a line defect in the SOS surface, created by a pair of $q$-fold screw dislocations at $\infty$ and $-\infty$ in the case of an infinite cylinder.

Conversely, we note that for an SOS configurations with an odd total height difference or equivalently with an odd number of strings, there must be an odd number of sign changes along the loop. This can be realized only in an Ising model with antiperiodic boundary conditions.

For a finite lattice with cylindrical boundary conditions an SOS surface with a defect line is the analog of a periodic surface with nonzero average tilt. Since in equilibrium the tilt should vanish in the thermodynamic limit, we shall restrict ourselves to configurations without defects for the calculation of the free energy. Critical exponents will be obtained by introduction of the defects discussed above and from correlation effects.

\section{A. Conformal anomaly and spin-wave exponents}

The exact solution of this model is given in Appendix A. As explained above, $L$ has to be a multiple of 3 to 
satisfy the requirement of an SOS surface with zero average tilt. Indeed, the exact solution depends on whether or not this condition is satisfied. If $L$ is even in addition, we can write Eq. (A10) as

$$
\lim _{M \rightarrow \infty} \frac{1}{M} \ln \operatorname{Pf} \underline{A}_{2}=\sum_{j=L / 6}^{5 L / 6} \ln \left(2 \sin \frac{\pi j}{L}\right) .
$$

Application of the Euler-Maclaurin summation formula leads to

$$
\lim _{M \rightarrow \infty} \frac{1}{M} \ln \operatorname{Pf} \underline{A}_{2}=\frac{2 L}{\pi} \int_{0}^{\pi / 3} d x \ln (2 \cos x)-\frac{x \sqrt{3}}{6 L}+\cdots,
$$

while expansion of the summand of Eq. (A13) in $1 / L$ yields

$$
\begin{aligned}
\lim _{M \rightarrow \infty} & \frac{1}{M} \ln \left[\operatorname{Pf} \underline{A}_{3} / \operatorname{Pf} \underline{A}_{2}\right] \\
= & \frac{\pi^{2}}{8 L^{2}} \sum_{j=(L+6) / 6}^{5 L / 6} \sin ^{-2} \frac{\pi(2 j-1)}{2 L}+\cdots \\
= & \frac{\pi}{8 L} \int_{\pi / 6}^{5 \pi / 6} d x \sin ^{-2} x+\cdots \\
= & \frac{\sqrt{3} \pi}{4 L}+\cdots .
\end{aligned}
$$

The ellipses in Eqs. (13) and (14) stand for odd powers $(\geq 3)$ in $1 / L$ only. Each row of spins has a height $\xi=\sqrt{3} / 2$. Dividing the sum of Eqs. (13) and (14) by this factor we obtain the reduced free energy per unit of length $F(L)$ as a series depending only on odd powers of $L$ :

$$
\begin{aligned}
-F(L) & =\lim _{M \rightarrow \infty} \frac{2}{\sqrt{3} M} \ln \operatorname{Pf} \underline{A}_{3} \\
& =\frac{4 L}{\sqrt{3} \pi} \int_{0}^{\pi / 3} d x \ln (2 \cos x)+\frac{\pi}{6 L}+\cdots .
\end{aligned}
$$

The conformal anomaly $c$ may be obtained from the free energy $F(L)$ per unit length, with the general, large$L$ expression derived from conformal invariance, ${ }^{12,13}$ viz.,

$$
F(L) \simeq L f_{\infty}-\frac{\pi c}{6 L},
$$

where $f_{\infty}$ is the reduced bulk free-energy density. Comparison of Eqs. (15) and (16) shows that

$$
c=1 \text {. }
$$

In the Ising model, the staggered magnetization is the operator associated with the correlation function which decays most slowly with distance. Its anomalous dimension $X_{6}^{(s)}$ can be obtained from the asymptotic large- $M$ behavior of $\frac{1}{2} \operatorname{Pf} \underline{A}_{2}$ in the partition function, Eq. (A1). According to Eq. (44), this correction decays asymptotically exponentially with $M$. Denoting the exponential factor by $\exp \left[-M \zeta / \xi_{6}^{(s)}(L)\right]$, we obtain the correlation length $\xi_{6}^{(s)}(L)=2 L / \pi$. According to the theory of conformal invariance, the finite-size amplitude of a general correlation length $\xi(L)$ is related to the anomalous dimension $X$ of the associated operator ${ }^{14}$ according to

$$
X \simeq \frac{L}{2 \pi \xi(L)} .
$$

Using this relation we obtain

$$
X_{6}^{(s)}=\frac{1}{4} \text {. }
$$

This exponent agrees with the known value of the magnetic exponent calculated by Stephenson. ${ }^{2}$

The finite-size behavior for $L$ equal to odd multiples of 3 is similar, and leads to identical results for Eqs. (17) and (19). Finally we mention that the exact solution in the presence of a periodic modulation of the Ising couplings, corresponding with a $p=3$ spin-wave term in SOS language, ${ }^{3}$ reveals an Ising-like singularity in the free energy, so that

$$
X_{3}^{(s)}=1
$$

which is in agreement with the energylike (four-spin) correlation function exponent calculated by Stephenson. ${ }^{2}$

\section{B. Vortex exponents}

As mentioned above and illustrated in Fig. 1, a temperature-induced excitation in the present Ising model corresponds to a screw dislocation, i.e., the creation or annihilation of a pair of strings. Since each string corresponds to a step of three height units in the SOS language [see Eq. (11)], such an excitation corresponds to a six-fold vortex or antivortex. In an infinite system the correlation function $G_{q}^{(v)}(r)$ describing the probability of such a pair of opposite charges at a distance $r$ decays as

$$
G_{q}^{(v)}(r) \sim r^{-2 X_{q}^{(v)}},
$$

where the Gaussian model prediction, corresponding to $H=0$ in Eq. (1), for $X_{q}^{(v)}$ is given by Eq. (7).

Here we present an independent calculation of this exponent as a function of $q$. As a first step, the infinite two-dimensional plane is mapped onto a cylinder with circumference $L$. Covariance under this conformal mapping $^{14}$ implies that the correlation function $g_{L}(l)$ on the cylinder, where $l$ is the coordinate in the length direction of the cylinder, behaves as

$$
g_{L}(l) \sim \exp \left[-l / \xi_{q}^{(v)}(L)\right] \quad(l \gg L),
$$

where the correlation length $\xi_{q}^{(v)}(L)$ is related to $X_{q}^{(v)}$ by Eq. (18). The correlation function on the cylinder can be written as

$$
g_{L}(l)=\lim _{M \rightarrow \infty} \frac{Z_{q}^{(M)}(l)}{Z_{0}^{(M)}},
$$

where $Z_{q}^{(M)}(l)$ is the partition function evaluated in the presence of a pair of conjugate $q$ vortices with separation l. For vortices with a separation large on the scale of the width of the system we thus have

$$
g_{L}(l) \sim \exp \left\{l\left[F_{0}(L)-F_{q}(L)\right]\right\} \quad(l \gg>L),
$$

where

$$
F_{q}(L)=-\lim _{M \rightarrow \infty} \frac{2 \sqrt{3}}{3 M} \ln Z_{q}^{(M)}
$$


is the reduced free energy per unit of length of a system on a cylinder with a screw defect line of strength $q$. Combination of Eqs. (18), (22), and (24) yields

$$
X_{q}^{(v)} \simeq \frac{L\left[F_{q}(L)-F_{0}(L)\right]}{2 \pi} .
$$

We follow two different ways to study systems with $q \neq 0$. First we consider an ensemble in which $q$ is fixed at a nonzero value. This is automatically realized for systems with sizes that are not multiples of 3 . Second, one can generalize the Hamiltonian [Eq. (1)] by introducing anisotropy, the thermodynamic variable conjugate to the tilt of the SOS surface.

For $L=3 n \pm 1$ with $n$ integral and a system with $N_{s}$ strings one has $q=3 N_{s}-6 n \pm 2$, so that $q= \pm 2$ is the closest one can get to the equilibrium of zero tilt. In order to derive the free energy $F_{2}(L)$, we apply the EulerMaclaurin summation formula, using Eqs. (A10) and (A23), and expand the boundary terms in $1 / L$ :

$$
\begin{aligned}
\lim _{M \rightarrow \infty} \frac{1}{M} \ln Z_{2}^{(M)}= & \frac{2 L}{\pi} \int_{0}^{\pi / 3} d x \ln (2 \cos x)-\frac{\pi \sqrt{3}}{36 L} \\
& \mp \frac{5 \pi^{2}}{81 L^{2}}+\cdots,
\end{aligned}
$$

when $L=3 n \pm 1$, i.e.,

$$
\begin{aligned}
-F_{2}(L) & =\lim _{M \rightarrow \infty} \frac{2}{\sqrt{3} M} \ln \operatorname{Pf} \underline{A}_{3} \\
& =\frac{4 L}{\sqrt{3} \pi} \int_{0}^{\pi / 3} d x \ln (2 \cos x)-\frac{\pi}{18 L}+\cdots .
\end{aligned}
$$

Substitution in Eq. (26) of this result and that of Eq. (15) yields

$$
X_{2}^{(v)}=\frac{1}{9},
$$

in agreement with Eq. (7) with $q=2$ and $T_{R}=18$. For odd system sizes that are not multiples of 3, we skip the details, and mention only that the result Eq. (29) is reproduced.

Thus far we have considered the tilt $q$ (or equivalently the number of strings or pairs of opposite spins) as the independent thermodynamic variable. Alternatively, we can introduce a tilt by the conjugate independent variable $\epsilon$, defined such that the Ising coupling in the horizontal direction is $K+\epsilon$, and $K$ in the other two directions. We denote by $\widetilde{F}(\epsilon)$ the associated reduced free enthalpy per unit length of a strip of width $L$.

Disregarding the infinite contribution due to $K$ and finite-size corrections we have the following exact expression: ${ }^{4}$

$$
-\widetilde{F}(\epsilon)=\frac{2 \sqrt{3} L \epsilon}{3}+\frac{\sqrt{3} L}{3 \pi} \int_{0}^{\omega_{c}} d \omega\{\ln [2(1+\cos \omega)]-4 \epsilon\},
$$

with

$$
\cos \omega_{c}=\frac{1}{2} \exp (4 \epsilon)-1 .
$$

Second-order expansion in $\epsilon$ yields

$$
\widetilde{F}(\epsilon)=F(0)+\frac{2 \sqrt{3} L \epsilon}{9}-\frac{8 L \epsilon^{2}}{3 \pi}+\cdots .
$$

The reduced energy $E(\epsilon)$ per unit of length is equal to $-\epsilon d \widetilde{F} / d \epsilon$ or

$$
E(\epsilon)=-\frac{2 \sqrt{3} L \epsilon}{9}+\frac{16 L \epsilon^{2}}{3 \pi}+\cdots .
$$

However, the reduced energy per unit length can also be calculated directly from the number of strings: Each horizontal bond carries an energy $-\epsilon$ if intersected by a string, and $\epsilon$ otherwise. For a system with $N_{s}=\frac{2}{3} L+a \epsilon L$ strings, we thus have

$$
E(\epsilon)=-\frac{2 \sqrt{3} L \epsilon}{9}-\frac{4 \sqrt{3} a L \epsilon^{2}}{3}+\cdots,
$$

so that $a=-4 \sqrt{3} / 3 \pi$. The relation between the free energies is $F_{q}=\widetilde{F}(\epsilon)+E(\epsilon)$, and it follows that

$$
F_{q}(L)-F_{0}(L)=\frac{8 L \epsilon^{2}}{3 \pi}+\cdots,
$$

where $q=-(4 / \pi) \sqrt{3} L \epsilon$. Substitution in Eq. (26) gives

$$
X_{q}^{(v)}=\frac{q^{2}}{36}
$$

in agreement with Eq. (7) for the known value $T_{R}=18$, the Gaussian temperature of the $H=0$ Ising model, Eq. (1).

This derivation uses the bulk free energy, and thus it neglects the "conformal" finite-size contribution to the free energy, which is equal to $\pi /(6 L)$ per unit of length ${ }^{12}$ for systems with central charge $c=1$. This is inconsequential if these contributions, which appears in both free energies in (26), are equal and thus cancel. Indeed, calculation on the Gaussian mode ${ }^{12}$ show that the finite-size amplitude of the free energy consists of two independent parts: One is equal to $\pi /(6 L)$ and is identified as the conformal contribution of the unperturbed Gaussian model, while the other is introduced by a "seam" [equivalent to a tilted SOS surface and with the same tilt dependence as Eq. (35)]. This separation of amplitudes is furthermore in line with the derivation of Eq. (29).

\section{NUMERICAL FINITE-SIZE SCALING ANALYSIS}

The analytic results presented in the preceding section are restricted to the soluble case, the Ising model in a vanishing magnetic field, i.e., $H=0$ in Eqs. (1) and (2). We apply numerical finite-size scaling to the free energy and correlation lengths to investigate the $H \neq 0$ properties. The finite-size data are obtained by means of the transfer matrix $\underline{T}$ defined in Appendix B. The transfer matrix applies to a system with a cylinder geometry: infinitely long in one direction and periodic with finite size $L$ in the other direction. The calculations were performed up to $L=27$, corresponding with a transfer matrix of size $9373650^{2}$.

Expecting slow convergence because of logarithmic corrections at the Kosterlitz-Thouless transition, we are interested in as wide as possible a range of system sizes. Therefore we have chosen not to use the Ising spin repre- 
sentation which carries redundant information. Instead, we represent the ground state by nonintersecting strings; ${ }^{4}$ details of the construction of a transfer matrix using this representation are explained in Appendix B.

Since the number of strings, $N_{s}$, i.e., the number of pairs of opposite spins, per row is conserved, the transfer matrix decomposes into diagonal blocks or sectors associated with different values of $N_{s}$. The eigenvalues of the transfer matrix can therefore be labeled by $q$ via Eq. (11). Within blocks the eigenvalues used below may be associated with different spin-wave operators. We denote them by $\Lambda_{q}^{p}(L)$. With this notation $\Lambda_{q}^{0}(L)$ is the dominant eigenvalue of block $q$, the $q=0$ block containing the overall dominant eigenvalue.

From these eigenvalues one can calculate the reduced free energy per unit length,

$$
F_{q}(L)=-\frac{1}{3} \sqrt{3} \ln \Lambda_{q}^{0}(L) .
$$

The transfer matrix defined in Appendix B adds two rows of spins to the lattice. This accounts for the factor of 2 difference in the prefactors of Eqs. (25) and (37). Gaps in the transfer-matrix spectrum yield correlation lengths as usual. For the $q=0$ block the correlation length is given by

$$
\frac{1}{\xi_{p}^{(s)}(L)}=\frac{1}{3} \sqrt{3} \ln \frac{\Lambda_{0}^{0}(L)}{\Lambda_{0}^{p}(L)} .
$$

\section{A. The Kosterlitz-Thouless transition}

The Kosterlitz-Thouless (KT) phase transition can be located using the condition that $X_{2}^{(s)}$ is marginal at the transition. However, the smallest gap of the transfer matrix tends to have the best numerical convergence properties and this gap is associated with the $p=6$ spin-wave operator. With Eq. (6), the condition of marginality translates to $X_{6}^{(s)}=\frac{2}{9}$. Using Eq. (18), we can obtain finite-size estimates of the critical field $H_{c}$ by numerically solving for $H$ in the equation

$$
\xi_{6}^{(s)}(H, L)=\frac{9 L}{4 \pi} .
$$

Because of corrections to scaling, the solution, denoted $H(1, L)$, depends on the finite size $L$. Numerical data are given in Table $I$ for sizes $L=3, \ldots, 27$. These results do not appear to converge rapidly. Indeed, one expects logarithmic corrections due to the presence of a marginally irrelevant field. Integrating the renormalization equations (see, e.g., Nauenberg and Scalapino ${ }^{15}$ and Blöte and Nightingale ${ }^{16}$ ) including such a field and a finite-size field $L^{-1}$ leads to corrections with a form given by

$$
H(1, L)=H_{c}+a /(b+\ln L)+\cdots .
$$

We generated sequences of iterated estimates of $H_{c}$ by solving for $H(2, L), a(L), b(L)$, and $c(L)$ in the equations

$H(2, L)=H(1, l)+a(L) /[b(L)+\ln l]+c(L) / l^{2}$,

where $l=L, L+3, L+6$, and $L+9$. The last term in Eq. (4.1) accounts for a possible irrelevant exponent with
TABLE I. Estimates of the critical field $H_{c}$ at which the KT transition to the ordered Ising state takes place. Solutions $H(1, L)$ of Eq. (39) were obtained for finite sizes up to $L=27$. Iterated fits $H(2, L)$ (last column) seem to converge more rapidly.

\begin{tabular}{rcr}
\hline \hline$L$ & $H(1, L)$ & $H(2, L)$ \\
\hline 3 & 0.098117618145143 & 0.2843 \\
6 & 0.339112158002366 & 0.2695 \\
9 & 0.362806739565371 & 0.2666 \\
12 & 0.366650325704477 & 0.2676 \\
15 & 0.366070496281102 & 0.2672 \\
18 & 0.364319050913208 & 0.2662 \\
21 & 0.362309008314749 & \\
24 & 0.360330661076380 & \\
27 & 0.358476170229427 & \\
\hline
\end{tabular}

value -2 . The iterated estimates thus obtained are also shown in Table I. Although the variation in the latter results is rather small, the apparent convergence is not monotonic. Under these circumstances, we can only guess that the difference of the last estimate $H(2,18)$ and $H_{c}$ does not exceed by a factor greater than ten the difference $H(2 ; 15)-H(2,18)$ between the last two estimates. Thus, on the basis of the data in Table I we estimate $H_{c}=0.266 \pm 0.010$.

\section{B. Conformal anomaly}

Having numerically calculated $F_{0}(L)$ for a range of $L$ values, we can use Eq. (16) to determine the conformal anomaly $c$. Two subsequent finite-size results for the free energy are used to produce an estimate $c(1, L)$ of $c$, by solving for $c(1, L)$ and $f_{\infty}(L)$ in

$$
F_{0}(l)=l f_{\infty}(L)-\frac{\pi c(1, L)}{6 l},
$$

for $l=L$ and $l=L+3$. Corrections to scaling are given by power laws for critical systems, so that up to the Kosterlitz-Thouless transition it is reasonable to introduce iterated estimates by solving for $c(2, L), a(L)$, and $b(L)$ in

$$
c(1, l)=c(2, L)+a(L) l^{-b(L)},
$$

with $l=L, l=L+3$, and $l=L+6$. This process can be iterated to yield $c(3, L)$; etc., until the process terminates because of round-off errors or lack of data. This procedure was applied to the free energies of systems up to size $L=24$ for $H=0,0.1,0.2,0.3$, and 0.4 . The results are shown in Table II, together with the estimated uncertainties in the last decimal place. These results demonstrate that the conformal anomaly stays approximately constant as $H$ varies.

\section{Exponents}

The vortex exponents $X_{q}^{(v)}$ can be calculated from Eq. (26). By a minor generalization of this expression one can calculate the exponent $X_{q}^{(v)}=\frac{1}{3}$ from the difference $F_{ \pm 4}(3 n \pm 1)-F_{\mp 2}(3 n \pm 1)$, where the two terms are calculated from systems containing $2 n \pm 2$ and $2 n$ strings, respectively, at $H=0$. We have confirmed this value numerically, but the extrapolation revealed strong correc- 
TABLE II. Estimates of the conformal anomaly $c$ and the anomalous dimensions $X_{6}^{(s)}, X_{3}^{(s)}$, and $X_{2}^{(v)}$. Estimated numerical inaccuracies in the last decimal place are given between parentheses.

\begin{tabular}{cccccl}
\hline \hline Quantity & $H=0$ & $H=0.1$ & $H=0.2$ & $H=0.3$ & $H=0.4$ \\
\hline$c$ & $1.000000(1)$ & $1.0000(1)$ & $1.0003(3)$ & $1.001(1)$ & $0.999(2)$ \\
$X_{6}^{(s)}$ & $0.250000(1)$ & $0.2479(1)$ & $0.2410(5)$ & $0.228(4)$ & $0.20(1)$ \\
$X_{3}^{(s)}$ & $1.000000(2)$ & $0.991(2)$ & $0.96(1)$ & $0.91(3)$ & $0.82(5)$ \\
$\boldsymbol{X}_{2}^{(v)}$ & $0.111111(1)$ & $0.1119(2)$ & $0.116(1)$ & $0.123(2)$ & $0.133(3)$ \\
$\boldsymbol{X}_{6}^{(s)} / X_{3}^{(s)}$ & $0.250000(2)$ & $0.2502(6)$ & $0.251(3)$ & $0.25(1)$ & $0.24(3)$ \\
$\boldsymbol{X}_{2}^{(v)} \boldsymbol{X}_{3}^{(s)}$ & $0.111111(1)$ & $0.1110(2)$ & $0.111(2)$ & $0.112(6)$ & $0.109(9)$ \\
\hline \hline
\end{tabular}

tions to scaling proportional to $1 / L$. These corrections, which are unusual (in most cases terms dominate effectively behaving as $L^{-2}$ ) are due to the presence of a $L^{-2}$ term in $F(L)$ when $N_{s} \neq 2 L / 3$ - see Eq. (27). While such corrections are still tractable in the numerical analysis at $H=0$, the case $N_{s} \neq 2 L / 3$ is difficult to analyze for $H \neq 0$.

In view of this problem we have chosen to limit ourselves to quantities that can be calculated with $N_{s}=2 L / 3$ and the behavior of the free energy as a function of $L$. That is, the average $\bar{F}_{2}(L)=\frac{1}{2} F_{2}(L-1)+\frac{1}{2} F_{-2}(L+1)$, where $L$ is a multiple of 3 , can be used to approximate $F_{2}(L)$, a quantity that cannot be calculated directly. The difference $\bar{F}_{2}(L)-F_{0}(L)$ can then be used with Eq. (26) to obtain an effective exponent approximating $X_{2}^{(v)}$.

Figure 2(a) shows the behavior of this effective exponent as a function of the magnetic field $H$ for $L=3-18$. For $H=0$, the data agree well with the exact result (see Sec. II) $X_{2}^{(v)}=\frac{1}{9}$. For $H \neq 0$ the exponent increases, in agreement with the hypothesis ${ }^{3}$ that the corresponding Gaussian coupling increases with increasing $H$ up to the roughening transition at $T_{R}=16$. The expected value of the exponent at the transition point is $X_{2}^{(v)}=\frac{1}{8}$. Indeed, the data points seem reasonably convergent for $X_{2}^{(v)} \lesssim \frac{1}{8}$. At higher values of $H$, crossover to a different behavior takes place associated with a flat phase in the SOS model in which $X_{2}^{(s)}$ is relevant.

The anomalous dimension of spin-wave operators can be estimated from corresponding correlation lengths as determined from subsequent eigenvalues in the $N_{s}=2 L / 3$ sector of the transfer matrix. The secondlargest eigenvalue is the dominant eigenvalue of the $q=0$ sector of states that are antisymmetric under spin global inversion at $\underline{H}=0$. This state is associated with the Ising spin-spin correlation function, which has an exponent denoted by $X_{6}^{(s)}$. The estimate $X_{6}^{(s)}(1, L)$ is obtained from Eq. (18) for subsequent system sizes, in analogy with Eq. (42). Numerical results for $X_{6}^{(s)}(1, L)$, which were already obtained in Ref. 11, are included in Fig. 2(b) for comparison. They illustrate the dual behavior of spin-wave and vortex exponents: Their values are, at least approximately, inversely proportional, as they should according to Eqs. (6) and (7). Both sets of data points agree with the presence of a Kosterlitz-Thouless transition near $H \approx 0.3$.

In addition to $X_{2}^{(v)}$ and $X_{6}^{(s)}$ we have also investigated $X_{3}^{(s)}$ as a function of $H$. The latter is obtained from the second-largest eigenvalue of the $q=0$ sector of states symmetric under spin inversion at $\underline{H}=0$. The results, ob- tained from iterated fits assuming power-law corrections, are summarized in Table II. The latter dimension is associated with the third- or fourth-largest eigenvalue of the $N_{s}=2 L / 3$ sector of the transfer matrix, depending on $L$ and $H$. The crossing eigenvalue was analyzed only in the case $H=0$; the corresponding eigenstate is antisymmetric under spin inversion. The fits are less well behaved and the associated dimension $X=1.25 \pm 0.01$ has only three significant decimal places, in contrast with the six or seven for $c$ and the other anomalous dimensions for $H=0$.

While the exponents vary continuously with $H$, Eqs. (6) and (7) imply that ratios of spin-wave or vortex exponents, and the product of vortex and spin-wave exponents are constants. Indeed, Table II illustrates this behavior. It is remarkable that the renormalizationgroup predictions still seem to apply for $H=0.3$ and 0.4 , which is outside the algebraic phase. As quantitative results they are pointless; however, they provide an illustration of the anomalously slow crossover behavior in the ordered phase near a roughening transition (i.e., in the disordered phase near a Kosterlitz-Thouless transition).

Finally, the numerical estimates of the location of the Kosterlitz-Thouless transition were based on the prediction $X_{6}^{(s)}=\frac{2}{9}$, i.e., $T_{R}=16$. From Eq. (7) it follows that at this point one should have $X_{2}^{(v)}=\frac{1}{8}$. To demonstrate the consistency of our estimate of the transition point we display the numerical estimates of $X_{2}^{(v)}$ obtained at $H=0.266$ in Table III. Two estimates are given: one derived directly from Eq. (18); the other was obtained by an iterated fit assuming $1 / \ln L$ corrections.

TABLE III. Estimates of the anomalous dimension $X_{2}^{(v)}$ at the field-induced KT transition. The iterated fits yielding $X_{2}^{(v)}(2, L)$ make use of the expected scaling behavior at the KT transition which is of the form $X_{2}^{(v)}(1, L)=X_{2}^{(v)}$ $+s /(t+\ln L)+\cdots$; this is different from that at other critical points.

\begin{tabular}{rcc}
\hline \multicolumn{1}{l}{$\boldsymbol{L}$} & $X_{2}^{(v)}(1, L)$ & $X_{2}^{(v)}(2, L)$ \\
\hline 3 & 0.0807968460 & 0.1431 \\
6 & 0.1048978632 & 0.1382 \\
9 & 0.1110443178 & 0.1327 \\
12 & 0.1135484356 & 0.1292 \\
15 & 0.1148347334 & 0.1269 \\
18 & 0.1155976968 & 0.1255 \\
21 & 0.1160970293 & 0.1244 \\
24 & 0.1164479707 & \\
\hline \hline
\end{tabular}



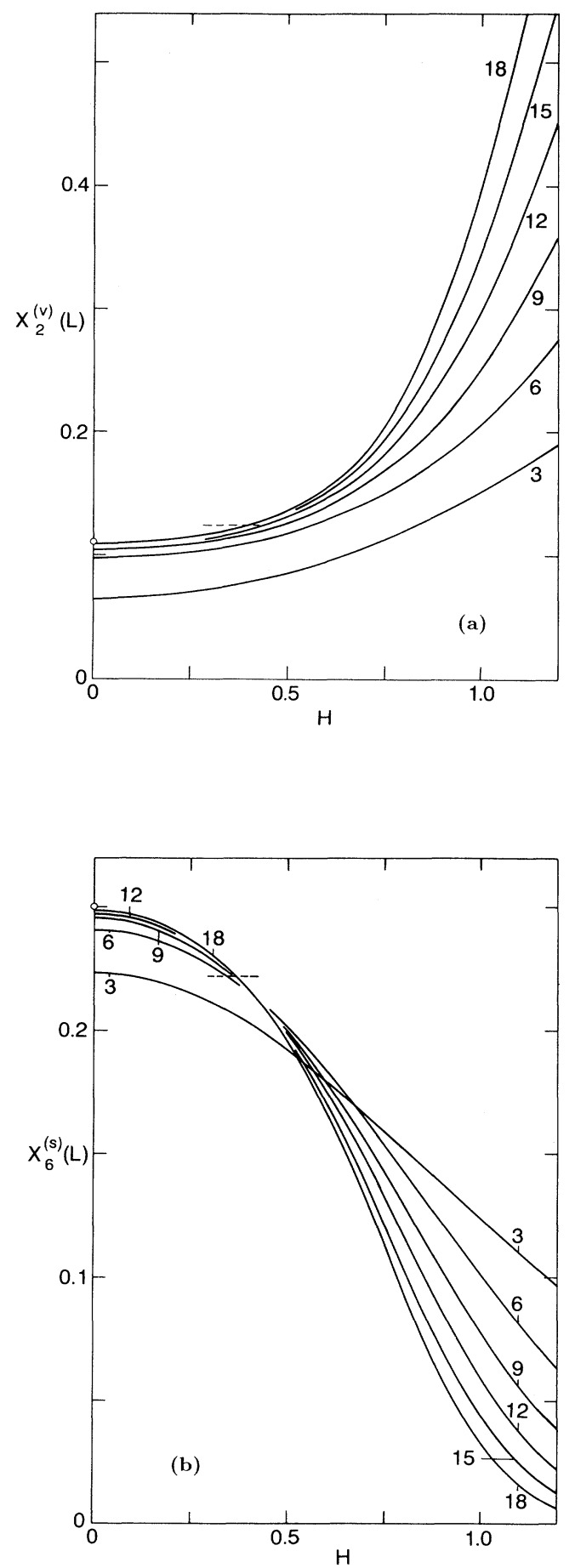

FIG. 2. Transfer-matrix results for the effective exponent associated with (a) vortices of strength 2 and (b) spin waves of period 6 vs the Ising magnetic field $H$. The data points (not shown) are connected by smooth curves labeled by the system size $L$. The data for both exponents at $H=0$ converge rapidly to the exactly known values (indicated as $O$ ). The short dashed lines indicate the predicted values of the exponents at the expected KT-like transition. Not shown are those parts of the $L=12$ and 15 curves that lie too close to the $L=18$ curve.

\section{DISCUSSION}

The mapping of the triangular zero-temperature Ising model onto the Gaussian model and the Coulomb gas involves a nonrigorous renormalization step. We summarize the following evidence that this step indeed is justified.

The renormalization-group scenario predicts that the amplitude of the height-height correlation function of the SOS mode is $A=T_{R} / 2 \pi^{2}$, that the anomalous dimension of the spin-spin correlation function is given by $X_{p}^{(s)}=T_{R} / 2 p^{2}$ with $p=6$, and that a periodic modulation of the Ising couplings as studied in Ref. 3 is described by the similar exponent with $p=3$. In zero magnetic field the predicted ratios agree with the previously known exact values $A=9 / \pi^{2},{ }^{4} X_{6}^{(s)}=\frac{1}{4},{ }^{2}$ and $X_{3}^{(s)}=1 .^{3}$

Likewise, the exact calculation of the general vortex exponent $X_{q}^{(v)}=q^{2} / 36$ in this paper confirms the prediction Eq. (7). At the same time the exact results reported here confirm the predicted relations between finite-size amplitudes and critical exponents. ${ }^{14}$ Similarly, in zero field, the exact result $c=1$ - see Eq. (17) -for the conformal anomaly confirms the conformal invariance prediction for models with continuously varying exponents. $^{17}$

For nonvanishing magnetic fields our numerical results show no deviations within the numerical accuracy from $c=1$ and also the appropriate ratios and products of exponents remain constant. This was verified in particular at the estimated Kosterlitz-Thouless transition point.

In addition to exponents that can be directly interpreted in terms of spin-wave and vortex operators, we have also found evidence for a scaling dimension $X=\frac{5}{4}$ at $H=0$. This value does not correspond to an integral spin-wave number. It could be a conformal follower ${ }^{18,19}$ of $X_{6}^{(s)}=\frac{1}{4}$.

The analytic and numerical results indicate that contributions to $F(L)$ proportional to $L^{-2}$ (i.e., of order $L^{-1}$ with respect to the leading finite-size dependence) arise whenever an excess number of strings is introduced. We propose an interpretation on the basis of anisotropic couplings in the context of the Gaussian model (different couplings between neighbors in the $x$ direction and those in the $y$ direction) to which the SOS model renormalizes. Note that $N_{s}=2 L / 3$ is a special symmetric case corresponding with a "horizontal" SOS surface. However, positive and negative slopes are not related by symmetry. An excess number of strings does not only introduce a slope in the surface (which can be transformed away in the Gaussian model), it also destroys the symmetry between the $x$ and $y$ directions. This deviation from symmetry is proportional to $1 / L$ for a given excess; this may explain the $1 / L$ corrections.

\section{ACKNOWLEDGMENTS}

We are much indebted to B. Nienhuis and M. J. Renne for valuable discussions, to $\mathrm{H}$. J. Hilhorst for his contributions to the earlier stages of this project, and to $\mathrm{X}$. N. $\mathrm{Wu}$, for independently reproducing a part of the finitesize data, thus providing a test of the algorithms. This 
research is supported by the National Science Foundation (NSF) through Grant No. DMR-87-04730 and by the NATO through Grant No. CRG 910152. The computations were performed in part at Cornell National Supercomputing Facility, a resource of the Center for Theory and Simulation in Science and Engineering at Cornell University, which is funded in part by the NSF, New York State, and the IBM Corporation. Part of this work was funded by the Dutch Foundation for "Fundamental Research on Matter (FOM)" which is financially supported by the "Nederlandse Organisatie voor Wetenschappelijk Onderzoek (NWO)."

\section{APPENDIX A: SOLUTION OF FINITE DIMER MODELS}

To calculate the partition function of the zerotemperature antiferromagnetic triangular Ising model, we make use of the equivalence with the dimer model on the honeycomb lattice. ${ }^{4}$ The correspondence between the rhombus tiling of the triangular lattice (and thus the string representation) and the dimer covering of the dual honeycomb lattice is illustrated in Fig. 3. Exact solutions of dimer models by means of Pfaffians were constructed by Kasteleyn ${ }^{20}$ and by Temperley and Fisher. ${ }^{21} \mathrm{~A}$ detailed review of the method was given by McCoy and $\mathrm{Wu}^{22}$ See also a more recent review of dimer models by Nagle, Yokoi, and Bhattacharjee. ${ }^{23}$

The residual entropy may be greatly reduced by boundary conditions. They can in fact be specified so as to completely remove the degeneracy of the ground state. To avoid this problem, we impose a toroidal topology on the system.

The honeycomb lattice is deformed into a rectangular grid with horizontal size $2 L$ and vertical size $M$. The labeling of the lattice sites by coordinates $1 \leq j \leq 2 L$ and $1 \leq k \leq M$ is shown in Fig. 4. Clearly there is a translational symmetry over two lattice units in the horizontal direction and over one unit in the vertical direction.

A first step of the Pfaffian solution involves the assignment of an orientation to each lattice edge that can be covered by a dimer. Four possible assignments are also shown in Fig. 4; the only differences occur between columns $2 L$ and 1 and between rows $M$ and 1 . A matrix $\underline{A}_{i}(i=1,2,3$, or 4$)$ is associated with each of the four
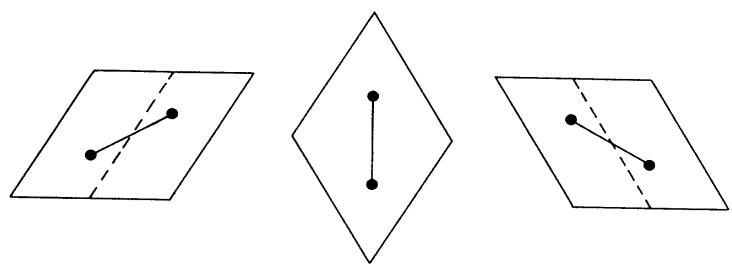

FIG. 3. Correspondence between the rhombus tiling of the triangular lattice and the dimer model on the honeycomb lattice. Rhombi consist of pairs of elementary faces, and can thus be represented by dimers (shown as dumbbells) covering a pair of sites on the dual, i.e., honeycomb lattice. String segments are indicated by dashed lines.

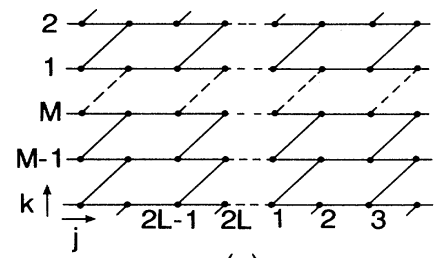

(a)

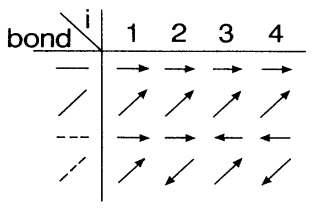

(b)
FIG. 4. Site labeling of the honeycomb lattice deformed to a square grid (a) and definition of the arrows for the four matrices $\underline{A}_{i}(\mathrm{~b})$.

choices for the edge orientations. The sites of the lattice are combined into pairs. The pair labeled $(j, k)$ contains the sites $(2 j-1, k)$ and $(2 j, k)$. A binary index $\alpha=1,2$ distinguishes the sites within each pair.

The absolute value of the matrix elements $\left|A\left(\alpha, j, k ; \alpha^{\prime}, j^{\prime}, k^{\prime}\right)\right|$ is equal to the weight of a dimer covering the bond between site $(\alpha, j, k)$ and $\left(\alpha^{\prime}, j^{\prime}, k^{\prime}\right)$. The weights are $z_{1}$ for the horizontal bonds between nearest-neighbor sites and $z_{2}$ for the diagonal bonds. We will be primarily interested in the case $z_{1}=z_{2}=1$. Since nonzero elements are associated only with nearestneighbor bonds, the $\underline{A}_{i}$ are sparse matrices. Furthermore, a nonzero matrix element $A\left(\alpha, j, k ; \alpha^{\prime}, j^{\prime}, k^{\prime}\right)$ is positive if the arrow-see Fig. 4-points from $(\alpha, j, k)$ to $\left(\alpha^{\prime}, j^{\prime}, k^{\prime}\right)$ and negative if it points the other way.

The derivation of the partition function $Z$ of the present Ising model wrapped on a torus differs somewhat from the derivation for the dimer model given in Ref. 22. That is, apart from dimer configurations for periodic Ising configurations, there are also those for antiperiodic Ising configurations. The number of changes of sign along a cyclic path crossing the periodic boundaries is even for the periodic case and odd for the case of antiperiodic boundary conditions. For $M$ even and equal to a multiple of $L$, a combination of Pfaffians in which the latter antiperiodic configurations are suppressed is

$Z=\frac{1}{2}\left[(-1)^{L}\left(\operatorname{Pf} \underline{A}_{1}+\operatorname{Pf} \underline{A}_{3}\right)+\operatorname{Pf} \underline{A}_{2}+\operatorname{Pf} \underline{A}_{4}\right]$

This can be understood by a direct application of the procedure described by McCoy and Wu in Chap. IV, Sec. 5 of Ref. 22. As noted before, only configurations with an even number of vertical strings are allowed for systems with periodic boundary conditions. Each allowed class of $C_{0}$ transition cycles (defined in Ref. 22) specified by the number of loops around the torus in the horizontal $h$ and vertical $v$ directions contributes twice (i.e., 4 times onehalf) to the linear combination of Pfaffians in Eq. (A1), once for each allowed Ising spin configuration. The disallowed classes contribute zero because the four contributions cancel. The latter classes are characterized by an odd number of strings or an odd number of windings over the vertical periodic boundary. The sign of the contributions to Eq. (A1) due to each Pfaffian is given in Table IV which is McCoy and Wu's table ${ }^{22}$ modified by the additional factors $(-1)^{L}$ in Eq. (A1). The sum of the four 
TABLE IV. Signs of the contributions of the four Pfaffians, due to configurations generated by $C_{0}$ transition cycles, to $Z$ in Eq. (A1).

\begin{tabular}{ccccc}
\hline \hline$(h, v)$ & $A_{1}$ & $A_{2}$ & $A_{3}$ & $A_{4}$ \\
\hline$(0,0)$ & $+(-1)^{L}$ & + & $+(-1)^{L}$ & + \\
$(o, e)$ & $-(-1)^{L}$ & - & $+(-1)^{L}$ & + \\
$(e, o)$ & $-(-1)^{L}$ & + & $-(-1)^{L}$ & + \\
$(o, o)$ & $-(-1)^{L}$ & + & $+(-1)^{L}$ & - \\
\hline
\end{tabular}

contributions due to a $C_{0}$ transition cycle with $(h, v)=(0,0)$ contributes only for even $L$. The class with $(h, v)=(2 n, 2 m-1)$, where $n$ and $m$ are natural numbers, only contributes for odd $L$, as it should since the number of strings is $L$ for dimer configurations in which only hor- izontal bonds are covered, and this number changes by one for each time an elementary transition cycle loops the torus in the vertical direction. The two remaining classes, which have $h=$ odd, correspond with Ising configurations that are antiperiodic in the vertical direction (note that $M$ is even), and their contributions cancel.

For the calculation of $Z$, it is convenient that the Pfaffians satisfy

$$
\operatorname{Pf} \underline{A}_{i}= \pm\left|\operatorname{det} \underline{A}_{i}\right|^{1 / 2},
$$

but the signs remain to be determined. Each pair $(j, k)$ contributes six nonzero elements to the $\underline{A}_{i}$ : Two are due to the internal bond, and four are due to bonds connecting to other pairs. Thus, the matrices $\underline{A}_{i}, i=1,2,3$, and 4 , can be written as

$\underline{A}_{i}=\left[\begin{array}{cc}0 & z_{1} \\ -z_{1} & 0\end{array}\right] \otimes \underline{I}_{L} \otimes \underline{I}_{M}+\left[\begin{array}{cc}0 & -z_{1} \\ 0 & 0\end{array}\right] \otimes \underline{H}_{i, L}^{T} \otimes \underline{I}_{M}+\left[\begin{array}{cc}0 & 0 \\ z_{1} & 0\end{array}\right] \otimes \underline{H}_{i, L} \otimes \underline{I}_{M}+\left[\begin{array}{cc}0 & 0 \\ -z_{2} & 0\end{array}\right] \otimes \underline{I}_{L} \otimes \underline{H}_{i, M}^{T}+\left[\begin{array}{cc}0 & z_{2} \\ 0 & 0\end{array}\right] \otimes \underline{I}_{L} \otimes \underline{H}_{i, M}$,

where the subscripts $L$ and $M$ serve to distinguish between the matrices acting on the row and column indices, respectively. We have used the definitions $I_{L}\left(j, j^{\prime}\right)=\delta_{j, j^{\prime}}$ and $H_{i, L}\left(j, j^{\prime}\right)=\delta_{j, j^{\prime}+1}+\left(1-2 p_{i}\right) \delta_{j, L} \delta_{j^{\prime}, 1}$ with $p_{1}=p_{2}=0$ and $p_{3}=p_{4}=1$. The definition of $\underline{I}_{i, M}$ is similar, as is that of $\underline{H}_{i, M}$, but with $p_{i}$ replaced by $q_{i}$ where $q_{1}=q_{3}=0$ and $q_{2}=q_{4}=1$. The indices of the $2 \times 2$ matrices label the spins within the pairs. After standard Fourier transformation one obtains

$$
\begin{aligned}
\operatorname{det} \underline{A}_{i}=\prod_{j=1}^{L} \prod_{k=1}^{M} & {\left[z_{1}\left(1-e^{i x_{i}(j)}\right)+z_{2} e^{-i y_{i}(k)}\right] } \\
& \times\left[z_{1}\left(1-e^{-i x_{i}(j)}\right)+z_{2} e^{i y_{i}(k)}\right],
\end{aligned}
$$

with

$$
\begin{aligned}
& x_{i}(j)=\pi\left(2 j-p_{i}\right) / L, \\
& y_{i}(k)=\pi\left(2 k-q_{i}\right) / M .
\end{aligned}
$$

The next step is to determine the signs in Eq. (A2). To this purpose we put $z_{1}=0$ so that the dimers are forced into pairs $(2 j-1, k ; 2 j, k+1)$. The sign of the Pfaffians then depends only on the vertical boundary condition specified by $i$ and on the parity of the permutation with respect to the reference configuration $(2 j-1, k ; 2 j, k)$. The result is that for $z_{1}=0$

$$
\begin{aligned}
& \operatorname{Pf} \underline{A}_{1}, \operatorname{Pf} \underline{A}_{3}<0 \quad(L \text { odd }), \\
& \operatorname{Pf} \underline{A}_{1}, \operatorname{Pf} \underline{A}_{3}>0 \quad(L \text { even }), \\
& \operatorname{Pf} \underline{A}_{2}, \operatorname{Pf} \underline{A}_{4}>0 \quad(\text { all } L) .
\end{aligned}
$$

Since the Pfaffians are analytic functions of $z_{1}$ and $z_{2}$, their signs for $z_{1}>0$ can be determined from the number of zeros when $z_{1}$ is varied. Each single zero in $\operatorname{Pf} \underline{A}_{i}$ corresponds, because of Eq. (A2), to a double zero of $\operatorname{det} \underline{A}_{i}$ and hence to single zero of $z_{1}\left(1-e^{i x_{i}(j)}\right)+z_{2} e^{-i y_{i}(k)}$ for some wave vector $(j, k)$. Such zeros may occur for special values of $z_{1}$-see Fig. 5-when $\pi-x_{i}(j)$ $=2\left[y_{i}(k)-\pi\right]$. However, they occur in pairs $\left(x_{i}, y_{i}\right)$ and $\left(2 \pi-x_{i}, 2 \pi-y_{i}\right)$, except when $x_{i}=y_{i}=\pi$. If such a single zero occurs, it is located at $z_{1}=z_{2} / 2$, and it is the only case in which a Pfaffian can change sign. It follows from Eq. (A5) that a change of sign occurs only in $\operatorname{Pf} \underline{A}_{3}$ for odd $L$ and in $\operatorname{Pf} \underline{A}_{1}$ for even $L$. Thus it follows from Eq. (A4) that, for the case $z_{1}=z_{2}=1$, to which we will restrict ourselves from now on,

$$
\begin{array}{r}
\operatorname{Pf} \underline{A}_{i}=r_{i} \prod_{j} \prod_{k}\left\{3-2 \cos x_{i}(j)+2 \cos y_{i}(k)\right. \\
\left.-2 \cos \left[x_{i}(j)+y_{i}(k)\right]\right\}^{1 / 2},
\end{array}
$$

with $-r_{1}=r_{2}=r_{3}=r_{4}=1$.

Next, we consider the behavior of the Pfaffians in the limit $M \rightarrow \infty$. If no zeros occur in Eq. (A7), it leads to

$$
\begin{aligned}
& \lim _{M \rightarrow \infty} \frac{1}{M} \ln \left(r_{i} \operatorname{Pf} \underline{A}_{i}\right) \\
&=\frac{1}{4 \pi} \sum_{j=1}^{L} \int_{0}^{2 \pi} d y \ln \left\{3-2 \cos x_{i}(j)+2 \cos y\right. \\
&\left.-2 \cos \left[x_{i}(j)+y\right]\right\},
\end{aligned}
$$

which can be rewritten as 


$$
\lim _{M \rightarrow \infty} \frac{1}{M} \ln \left(r_{i} \operatorname{Pf} \underline{A}_{i}\right)=\frac{1}{4 \pi} \sum_{j=1}^{L} \int_{0}^{2 \pi} d y \ln \left\{3-2 \cos x_{i}(j)-4 \sin \left[\frac{1}{2} x_{i}(j)\right] \sin \left[y+\frac{1}{2} x_{i}(j)\right]\right\} .
$$

Using $\int_{0}^{2 \pi} d x \ln (a+b \sin x)=2 \pi \ln \left[\frac{1}{2}\left(a \sqrt{a^{2}-b^{2}}\right)\right]$, it follows that

$$
\begin{aligned}
\lim _{M \rightarrow \infty} \frac{1}{M} \ln \left(r_{i} \operatorname{Pf} \underline{A}_{i}\right) & =\frac{1}{2} \sum_{j=1}^{L} \ln \left[\frac{1}{2}+2 \sin ^{2} \frac{x_{i}(j)}{2}+\left|\frac{1}{2}+2 \sin ^{2} \frac{x_{i}(j)}{2}\right|\right] \\
& =\sum_{j=1}^{L} \Theta\left[\sin \frac{x_{i}(j)}{2}-\frac{1}{2}\right] \ln \left\{2 \sin \frac{x_{i}(j)}{2}\right\},
\end{aligned}
$$

where $\Theta$ is the unit-step function. This result is the same for $i=1$ and 2 and also for $i=3$ and 4 . Thus the Pfaffians (if nonzero) form two approximately equal pairs.

Therefore, while evaluating Eq. (A1) in the limit $M \rightarrow \infty$, it may not be sufficient to select one of the largest Pfaffians since cancellations may occur of the contributions due to $\operatorname{Pf} \underline{A}_{1}$ and $\operatorname{Pf} \underline{A}_{2}$, as well as of those due to $\operatorname{Pf} \underline{A}_{3}$ and $\operatorname{Pf} \underline{A}_{4}$. We distinguish the following four cases.

\section{Case 1: $L$ an odd multiple of 3}

The contributions to Eq. (A1) due to $\operatorname{Pf} \underline{A}_{1}$ and $\operatorname{Pf} \underline{A}_{2}$ both are positive. Any differences become unimportant in the limit specified by Eq. (A10). Furthermore $\operatorname{Pf} \underline{A}_{3}=0$ due to a factor 0 associated with the wave numbers $j=(L+3) / 6$ and $k=2 M / 3$ in Eq. (A7), and $\operatorname{Pf} \underline{A}_{4}<-\operatorname{Pf} \underline{A}_{1}$. This can be seen as follows. As a consequence of Eq. (A10) we have

$$
\begin{aligned}
\lim _{M \rightarrow \infty} \frac{1}{M} \ln \left[-\operatorname{Pf} \underline{A}_{1} / \operatorname{Pf} \underline{A}_{4}\right] \\
=\sum_{j=(L+3) / 6}^{(5 L-3) / 6}\left[\ln \left\{2 \sin \frac{\pi j}{L}\right\}-\frac{1}{2} \ln \left\{2 \sin \frac{\pi(2 j-1)}{2 L}\right\}\right. \\
\left.-\frac{1}{2} \ln \left\{2 \sin \frac{\pi(2 j+1)}{2 L}\right\}\right] .
\end{aligned}
$$

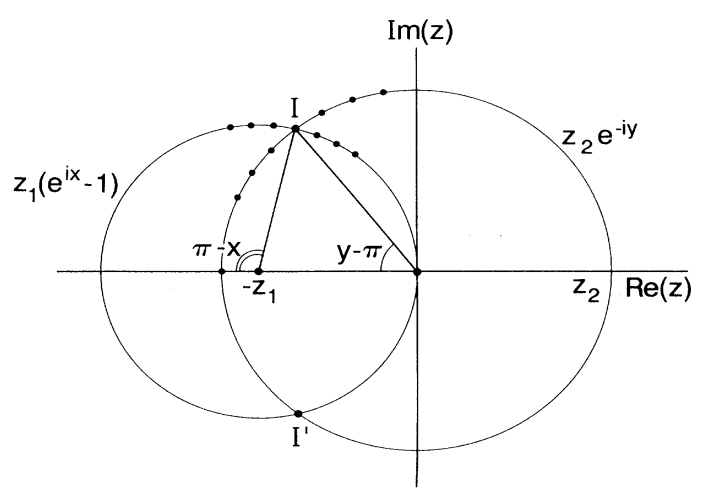

FIG. 5. Location of zeros of Eq. (A4) in the complex plane. Zeros occur for some value of $z_{1} / z_{2}$ if $\pi-x=2(y-\pi)$ (points $I$ and $I^{\prime}$ ). The phase angles $x$ and $y$ assume discrete values (indicated by $\bullet$ ) according to Eqs. (A5). Only in the case that $x=y=\pi$ (when $I$ and $I^{\prime}$ coincide) will this zero be accompanied by a change of sign of the Pfaffian. For $z_{1}=z_{2}$, a zero occurs for $x=\pi / 3, y=4 \pi / 3$ if allowed by Eqs. (A5).
Apparent deviations from Eq. (A10) concern only terms that vanish. Since $\ln (2 \sin x)$ is a convex function of $x$ in the pertinent interval, this expression is positive. It follows that

$$
\begin{array}{r}
\lim _{M \rightarrow \infty} \frac{1}{M} \ln Z=\lim _{M \rightarrow \infty} \frac{1}{M} \ln \left(-\operatorname{Pf} \underline{A}_{1}\right) \\
(L=6 n+3) .
\end{array}
$$

\section{Case 2: $L$ an even multiple of 3}

The contributions to Eq. (A1) due to $\operatorname{Pf} \underline{A}_{3}$ and $\operatorname{Pf} \underline{A}_{4}$ both are positive. Any differences become unimportant in the limit specified by Eq. (A10). Furthermore $\operatorname{Pf} \underline{A}_{1}=0$ due to a factor 0 associated with the same phase factors as in the case of odd $L$, and $\operatorname{Pf} \underline{A}_{2}<\operatorname{Pf} \underline{A}_{3}$. This is because

$$
\begin{aligned}
\lim _{M \rightarrow \infty} \frac{1}{M} \ln \left[\operatorname{Pf} \underline{A}_{3} / \operatorname{Pf} \underline{A}_{2}\right] & \\
= & \sum_{j=(L+6) / 6}^{5 L / 6}\left[\ln \left\{2 \sin \frac{\pi(2 j-1)}{2 L}\right\}\right. \\
& -\frac{1}{2} \ln \left\{2 \sin \frac{\pi(j-1)}{L}\right\} \\
& \left.-\frac{1}{2} \ln \left\{2 \sin \frac{\pi j}{L}\right\}\right]
\end{aligned}
$$

is positive for the same reason as in case 1 . It follows that

$$
\lim _{M \rightarrow \infty} \frac{1}{M} \ln Z=\lim _{M \rightarrow \infty} \frac{1}{M} \ln \left(\operatorname{Pf} \underline{A}_{3}\right) \quad(L=6 n) .
$$

\section{Case 3: $L$ odd and not a multiple of 3}

First we take $L$ equal to a multiple of 3 minus 1 . None of the Pfaffians is zero in this case. We check which pair of Pfaffians is the largest in absolute value: 


$$
\begin{aligned}
\lim _{M \rightarrow \infty} \frac{1}{M} \ln \left[-\operatorname{Pf} \underline{A}_{3} / \operatorname{Pf} \underline{A}_{1}\right]= & \sum_{j=(L+7) / 6}^{(5 L-1) / 6}\left[\ln \left\{2 \sin \frac{\pi(2 j-1)}{2 L}\right\}-\frac{1}{2} \ln \left\{2 \sin \frac{\pi j}{L}\right\}\right. \\
& \left.-\frac{1}{2} \ln \left\{2 \sin \frac{\pi(j-1)}{L}\right\}\right]-\ln \left\{2 \sin \frac{\pi(L+1)}{6 L}\right\} .
\end{aligned}
$$

Second-order approximation of the summand in $1 / L$ yields a positive contribution which dominates over the last term. Thus $\operatorname{Pf} \underline{A}_{3}$ and $\operatorname{Pf} \underline{A}_{4}$ are, in absolute value, larger than $\operatorname{Pf} \underline{A}_{1}$ and $\operatorname{Pf} \underline{A}_{2}$. But their contributions to Eq. (A1) have opposite signs. The Euler-Maclaurin summation formula applied to Eq. (A7) shows that all coefficients in the $1 / M$ expansion of $\ln \left[\operatorname{Pf} \underline{A}_{3} / \operatorname{Pf} \underline{A}_{4}\right]$ are zero and the possibility arises that the cancellation is so complete that the contributions due to $\operatorname{Pf} \underline{A}_{1}$ and $\operatorname{Pf} \underline{A}_{2}$ will dominate. For an analytic examination of this problem, a determination of the large- $M$ asymptotic behavior beyond that of Eq. (A10) is required. To this purpose we go back to Eq. (A4):

$$
\ln \left[-\operatorname{Pf} \underline{A}_{4} / \operatorname{Pf} \underline{A}_{3}\right]=\frac{1}{2} \sum_{j=1}^{L}\left(B_{j}+B_{j}^{*}\right)
$$

with

$$
B_{j}=\sum_{k=1}^{M} \ln \left[\frac{e^{-\pi i(2 k-1) / M}-\mu}{e^{-2 \pi i k / M}-\mu}\right],
$$

where $\mu=e^{\pi i(2 j-1) / N}-1$ satisfies $|\mu| \neq 1$. An equivalent definition is

$$
B_{j}=\frac{1}{2 i} \oint d z \cot (\pi z) \ln \left[\frac{e^{-\pi i(2 z-1) / M}-\mu}{e^{-2 \pi i z / M}-\mu}\right],
$$

because the cotangent has poles at integral values of $z$. The path of integration has to contain the integers $k=1,2, \ldots, M$ and no other singularities, i.e., associated with the points $z_{1}=(i M / 2 \pi) \ln \mu$ and $z_{2}=z_{1}+1 / 2$ where the numerator or the denominator of the argument of the logarithm vanishes. The associated branch cuts can be made to annihilate except on a line segment between $z_{1}$ and $z_{2}$. Next, the contour of integration is deformed into an elongated rectangle with its long dimension in the $\operatorname{Im}(z)$ direction. It is located at $1 / 2 \leq \operatorname{Re}(z) \leq M+\frac{1}{2}$ and $-y_{0} \leq \operatorname{Im}(z) \leq y_{0}$, while we consider the limit $y_{0} \rightarrow \infty$. The integration along the rectangle yields only an imaginary contribution that will eventually cancel. However, the branch cut is located inside the rectangle; integration along both sides of the cut yields, in the limit of large $M$,

$$
\operatorname{Re}\left(B_{j}\right)=2 \operatorname{Re}\left(\mu^{ \pm M}\right),
$$

where the \pm sign is such that $\left|\mu^{ \pm M}\right|<1$. The slowest decay in Eq. (A16) is associated with the wave number $j=(L+1) / 6$, for which $|\mu| \simeq 1-\pi / L \sqrt{3}$. Thus

$$
\left|\operatorname{Pf} \underline{A}_{4}-\operatorname{Pf} \underline{A}_{3}\right| \lesssim 2 e^{-\pi M / \sqrt{3} L} \operatorname{Pf} \underline{A}_{4} .
$$

This is to be compared with the contribution due to $\operatorname{Pf} \underline{A}_{2}$. From the foregoing it follows that also $\operatorname{Pf} \underline{A}_{2} / \operatorname{Pf} \underline{A}_{4}$ decays exponentially with $M$, but on a longer length scale $4 \sqrt{3} L / \pi$. Therefore, at least for sufficiently large $L$, the contributions due to $-\operatorname{Pf} \underline{A}_{1}$ and $\operatorname{Pf} \underline{A}_{2}$ dominate in the partition function when $M \rightarrow \infty$.

The case $L$ odd and equal to a multiple of 3 plus one is quite similar, and the answer is the same. Thus

$$
\lim _{M \rightarrow \infty} \frac{1}{M} \ln Z=\lim _{M \rightarrow \infty} \frac{1}{M} \ln \left(-\operatorname{Pf} \underline{A}_{1}\right) \quad(L=6 n \pm 1) .
$$

\section{Case 4: $L$ even and not a multiple of 3}

First we take $L$ equal to a multiple of 3 minus 1 . Then

$$
\begin{aligned}
\lim _{M \rightarrow \infty} \frac{1}{M} \ln \left[\operatorname{Pf} \underline{A}_{2} / \operatorname{Pf} \underline{A}_{4}\right]= & \sum_{j=(L+4) / 6}^{(5 L-4) / 6}\left[\ln \left\{2 \sin \frac{\pi j}{L}\right\}-\frac{1}{2} \ln \left\{2 \sin \frac{\pi(2 j-1)}{2 L}\right\}\right. \\
& \left.-\frac{1}{2} \ln \left\{2 \sin \frac{\pi(2 j+1)}{2 L}\right\}\right]-\ln \left\{2 \sin \frac{\pi(L+1)}{6 L}\right\} .
\end{aligned}
$$

In analogy with case 3 , this expression is positive. Thus $\operatorname{Pf} \underline{A}_{1}$ and $\operatorname{Pf} \underline{A}_{2}$ are largest in absolute value. They have opposite signs and their contributions nearly cancel in Eq. (A1), and it appears that $\operatorname{Pf} \underline{A}_{3}$ and $\operatorname{Pf} \underline{A}_{4}$ dominate. Since the case $L$ even and equal to a multiple of 3 plus one appears to behave similarly, the result is

$\lim _{M \rightarrow \infty} \frac{1}{M} \ln Z=\lim _{M \rightarrow \infty} \frac{1}{M} \ln \operatorname{Pf} \underline{A}_{3} \quad(L=6 n \pm 2)$.

\section{APPENDIX B: THE TRANSFER MATRIX}

The construction of the transfer matrix is based on the mapping of the zero-temperature antiferromagnetic triangular Ising model on a system of nonintersecting strings ${ }^{4}$ as described in Sec. I. Since the number $N_{s}$ of strings running in the length direction of the cylinder is conserved, the transfer matrix decomposes into sectors 
characterized by $N_{s}$. These strings intersect the horizontal edges between antiparallel spins and the other edges between parallel spins. Thus the positions of the strings are coded by bond variables $b_{i}$ located on the midpoints of the edges: $b_{i}=1$ describing the presence of a string and $b_{i}=0$ its absence. In order to determine the sign of the Ising spins we use, in addition to the bond variables, one Ising variable $s= \pm 1$.

The transfer-matrix construction involves establishing a one-to-one correspondence between the states of a row of spins and the positive integers $\alpha=1,2,3, \ldots$. There are $\left(\stackrel{L}{N_{s}}\right)$ ways in which positions of $N_{s}$ strings (i.e., the nonzero bond variables) can be distributed over $L$ edges. The enumeration of these distributions is straightforward and can be found, e.g., in Ref. 16. The additional Ising variable $s$ increases the number of states per row by a factor of 2. Thus, the state of row $M$ of the model is coded by an integer $1 \leq \alpha \leq N_{L}$ where $N_{L}=2\left({ }_{N_{s}}^{L}\right)$. Given $N_{s}$, the index $\alpha$ determines the spins thus denoted $s_{j}(\alpha)$ for the $j$ th site in the row.

The elements of the one-row transfer matrix $\underline{T}_{\text {row }}$ are

$$
\begin{aligned}
T_{\alpha \beta}^{\mathrm{row}}=\prod_{j=1}^{L} & \frac{1}{16}\left[3-s_{j}(\alpha) s_{j}(\beta)-s_{j}(\beta) s_{j+1}(\beta)\right. \\
& \left.-s_{j+1}(\beta) s_{j}(\alpha)\right] \\
& \times\left[3-s_{j}(\alpha) s_{j}(\beta)-s_{j}(\beta) s_{j-1}(\alpha)-s_{j-1}(\alpha) s_{j}(\alpha)\right] \\
& \times \exp \left\{\frac{1}{2} H\left[s_{j}(\alpha)+s_{j}(\beta)\right]\right\} .
\end{aligned}
$$

An infinite factor $\left(e^{-2 K}\right.$ per spin) due to the nearestneighbor coupling [see Eq. (1)] has been discarded.

Next we express $\underline{T}_{\text {row }}$ by means of the bond variables. The state $\alpha$ of the topmost row is denoted $\alpha=I\left(s, b_{1}, b_{2}, \ldots, b_{L}\right)$, where $I$ denotes the function whose value is the transfer-matrix index. After appending the new row, it is written $\alpha^{\prime}=I\left(s^{\prime}, b_{1}^{\prime}, b_{2}^{\prime}, \ldots, b_{L}^{\prime}\right)$. The transfer matrix becomes
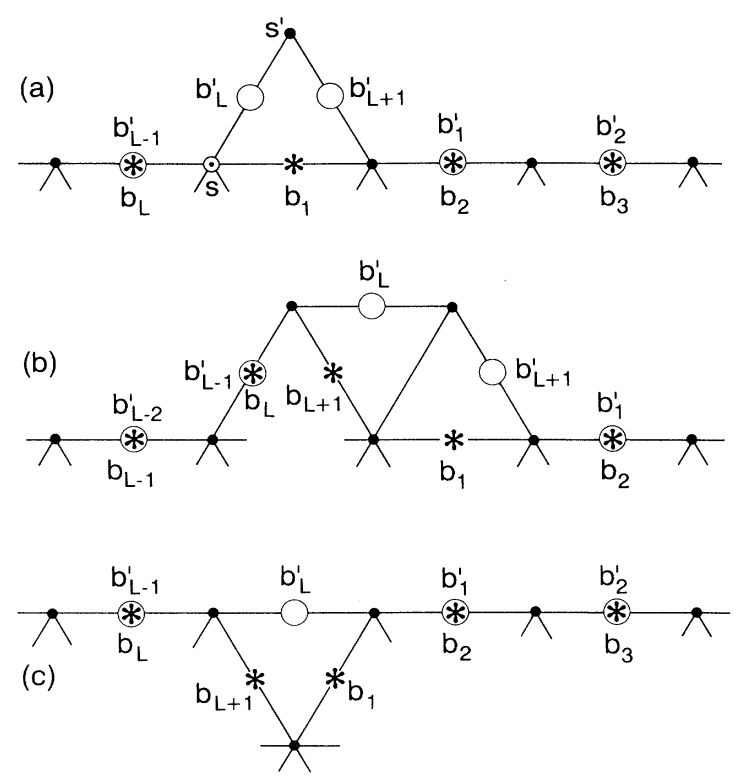

FIG. 6. Illustration of the action of the sparse transfer matrices $\underline{P}, \underline{Q}$ and $\underline{R}$, on the topmost boundary of a triangular lattice with periodic boundaries in the horizontal direction. The indices of these matrices correspond with states of the set of bond variables $b_{i}$ on the boundary edges supplemented with one spin value. The bond variables associated with the first matrix index are shown as $\bigcirc$, those with the second index as $*$. (a) The sparse matrix $\underline{R}$ appends the first site of a new row to the lattice. It thus increases the number of bond variables with one. Furthermore, it determines the new spin $(\bullet)$ on the basis of the old one $(\odot)$ and of the bond variable $b_{L}^{\prime}$. (b) This diagram shows $Q$ appending the second site of a new row, as evident from the shape of the boundary. However, Eq. (B6) applies also to appending sites 3 to $L$. (c) Multiplication by $\underline{P}$ reduces the number of boundary edges again to $L$. The last site was already appended by a $\underline{Q}$ matrix.

$$
T_{\alpha^{\prime}, \alpha}^{\mathrm{row}}=\left[T_{\alpha^{\prime}, \alpha^{\prime}}^{H}\right]^{1 / 2} \sum_{a_{1}=0}^{b_{1}} \sum_{a_{2}=0}^{b_{2}} \cdots \sum_{a_{L}=0}^{b_{L}} \delta_{s^{\prime}, s\left(2 a_{1}-1\right)} \prod_{j=1}^{L} \delta_{b_{k}^{\prime}, b_{k}\left(1-a_{k}\right)+b_{k+1} a_{k+1}}\left[T_{\alpha, \alpha}^{H}\right]^{1 / 2},
$$

where the $a_{i}$ describe whether a string meanders to the left $\left(a_{i}=1\right)$ or to the right $\left(a_{i}=0\right)$, and $\underline{T}_{H}$ is diagonal with elements

$$
T_{\alpha, \alpha}^{H}=\exp \left[s \sum_{k=1}^{L} \prod_{j=1}^{k-1}\left(2 b_{j}-1\right)\right] .
$$

For reasons of computational efficiency, $\underline{T}_{\text {row }}$ is decomposed into sparse matrices, in analogy with, e.g., Ref. 24 . By introducing powers of the shift operator $\underline{S}$ relabeling the bonds $b_{i} \rightarrow b_{i}^{\prime}=b_{i+1}(i=1,2, \ldots, L+1), L-1$ of these matrices become identical: ${ }^{16}$

$$
\underline{T}_{\text {row }}=\underline{T}_{H}^{1 / 2} \underline{P} \underline{Q}^{L-1} \underline{R T}_{H}^{1 / 2},
$$

where $\underline{R}=\underline{S T}_{1}, \underline{Q}=\underline{S}^{2} \underline{T}_{2} \underline{S}^{-1}$, and $\underline{P}=\underline{T}_{L+1} \underline{S}$ can be expressed as follows in bond variables:

$$
\begin{aligned}
& R\left(s^{\prime}, b_{1}^{\prime}, \ldots, b_{L+1}^{\prime} ; s, b_{1}, \ldots, b_{L}\right)=\delta_{b_{L}^{\prime}+b_{L+1}^{\prime}, b_{1}} \delta_{s^{\prime}, s\left(2 b_{L}^{\prime}-1\right)} \prod_{k=2}^{L} \delta_{b_{k-1}^{\prime}, b_{k}}, \\
& Q\left(s^{\prime}, b_{1}^{\prime}, \ldots, b_{L+1}^{\prime} ; s_{1} b_{1}, \ldots, b_{L+1}\right)=\left[\delta_{b_{L}^{\prime}+b_{L+1}^{\prime}, b_{L+1}+b_{1}}-b_{L+1}^{\prime} b_{L+1}\left(1-b_{L}^{\prime}\right)\left(1-b_{1}\right)\right] \delta_{s^{\prime}, s} \prod_{k=2}^{L} \delta_{b_{k-1}^{\prime}, b_{k}},
\end{aligned}
$$




$$
P\left(s^{\prime}, b_{1}^{\prime}, \ldots, b_{L}^{\prime} ; s, b_{1}, \ldots, b_{L+1}\right)=\delta_{b_{L}^{\prime}, b_{L+1}+b_{1}} \delta_{s^{\prime}, s} \prod_{k=2}^{L} \delta_{b_{k-1}^{\prime}, b_{k}} .
$$

The action of the sparse matrices $\underline{P}, \underline{Q}$, and $\underline{R}$ is illustrated in Fig. (6). Note the relabeling of the sites due to the shift operations.

For the determination of the largest eigenvalues, it is convenient to have a symmetric transfer matrix. The matrix

$$
\underline{T}=\underline{\widetilde{S}} \underline{T}_{\text {row }}^{2}
$$

where $\underline{\widetilde{S}}$ is the matrix representation of the shift operator relabeling the sites $s_{i} \rightarrow s_{i}^{\prime}=s_{i+1}(i=1,2, \ldots, L)$, satisfies this condition. Equation (B8) defines the transfer matrix used to derive the numerical data used in Sec. III. A number of eigenvalues can be obtained by application of the conjugate gradient algorithm ${ }^{25}$ in combination with orthogonalization and, where possible, imposing symmetries on the eigenvector. Since Eq. (B8) accounts for two layers of spins, each with a thickness $\xi=\frac{1}{2} \sqrt{3}$ lattice units, Eqs. (37) and (38) contain a factor $1 / 2 \xi$.

${ }^{1}$ R. F. M. Houtappel, Physica 16, 425 (1950).

${ }^{2}$ J. Stephenson, J. Math. Phys. A 11, 413 (1970).

${ }^{3}$ B. Nienhuis, H. J. Hilhorst, and H. W. J. Blöte, J. Phys. A 17, 2559 (1984).

${ }^{4}$ H. W. J. Blöte and H. J. Hilhorst, J. Phys. A 15, L631 (1982).

${ }^{5}$ J. V. José, L. P. Kadanoff, S. Kirkpatrick, and D. Nelson, Phys. Rev. B 16, 1217 (1977).

${ }^{6}$ H. J. F. Knops, Phys. Rev. Lett. 39, 766 (1977).

${ }^{7}$ B. Nienhuis, in Phase Transitions and Critical Phenomena, edited by C. Domb and J. L. Lebowitz (Academic, London, 1987), Vol. 11.

${ }^{8}$ M. P. M. den Nijs, in Phase Transitions and Critical Phenome$n a$, edited by C. Domb and J. L. Lebowitz (Academic, London, 1988), Vol. 12.

${ }^{9}$ J. M. Kosterlitz and D. J. Thouless, J. Phys. C 5, L124 (1973); 6, 1181 (1973).

${ }^{10}$ H. van Beijeren, Phys. Rev. Lett. 38, 993 (1977).

${ }^{11}$ H. W. J. Blöte, M. P. Nightingale, X. N. Wu, and A. Hoogland, Phys. Rev. B 43, 8751 (1991).

${ }^{12}$ H. W. J. Blöte, J. L. Cardy, and M. P. Nightingale, Phys. Rev. Lett. 56, 742 (1986).

${ }^{13}$ I. Affleck, Phys. Rev. Lett. 56, 746 (1986).

${ }^{14}$ J. L. Cardy, J. Phys. A 17, L358 (1984).
${ }^{15}$ M. Nauenberg and D. J. Scalapino, Phys. Rev. Lett. 44, 837 (1980).

${ }^{16}$ H. W. J. Blöte and M. P. Nightingale, Physica A 112, 405 (1982).

${ }^{17}$ J. L. Cardy, J. Phys. A 20, L891 (1987).

${ }^{18}$ A. A. Belavin, A. M. Polyakov, and A. B. Zamolodchikov, J. Stat. Phys. 34, 763 (1984).

${ }^{19}$ J. L. Cardy, in Phase Transitions and Critical Phenomena (Ref. 7).

${ }^{20}$ P. W. Kasteleyn, Physica 27, 1209 (1961); J. Math. Phys. 4, 287 (1963).

${ }^{21}$ H. N. V. Temperley and M. E. Fisher, Philos. Mag. 6, 1061 (1961).

${ }^{22}$ B. M. McCoy and T. T. Wu, The Two-Dimensional Ising Model (Harvard University Press, Cambridge, MA, 1973).

${ }^{23}$ J. F. Nagle, C. S. O. Yokoi, and S. M. Bhattacharjee, in Phase Transitions and Critical Phenomena, edited by C. Domb and J. L. Lebowitz (Academic, London, 1989), Vol. 13.

${ }^{24}$ H. W. J. Blöte, F. Y. Wu, and X. N. Wu, Int. J. Mod. Phys. C 4, 619 (1990).

${ }^{25}$ W. W. Bradbury and R. Fletcher, Numer. Math. 9, 259 (1966). 